\title{
Is diabetes in Cushing's syndrome only a consequence of hypercortisolism?
}

\author{
Carla Giordano, Valentina Guarnotta, Rosario Pivonello', Marco Calogero Amato, \\ Chiara Simeoli ${ }^{1}$, Alessandro Ciresi, Alessia Cozzolino' and Annamaria Colao' \\ Dipartimento di Medicina Interna e Specialistica (Di.Bi.Mi.S) Sezione di Endocrinologia e Malattie del Metabolismo \\ Università di Palermo, Piazza delle Cliniche 2, 90127 Palermo, Italy and ${ }^{1}$ Dipartimento di Medicina Clinica e \\ Chirurgia, Sezione di Endocrinologia, Università 'Federico II' di Napoli, Via S. Pansini 5, 80131 Naples, Italy
}

Correspondence
should be addressed
to C Giordano
Email
carla.giordano@unipa.it

\begin{abstract}
Objective: Diabetes mellitus (DM) is one of the most frequent complications of Cushing's syndrome (CS). The aim of this study was to define the changes in insulin sensitivity and/or secretion in relation to glucose tolerance categories in newly diagnosed CS patients.

Design: Cross-sectional study on 140 patients with CS.

Methods: A total of 113 women ( 80 with pituitary disease and 33 with adrenal disease, aged $41.7 \pm 15.7$ years) and 27 men (19 with pituitary disease and eight with adrenal disease, aged $38.1 \pm 20.01$ years) at diagnosis were divided according to glucose tolerance into normal glucose tolerance (CS/NGT), impaired fasting glucose and/or impaired glucose tolerance (CS/prediabetes), and diabetes (CS/DM) groups.

Results: Seventy-one patients had CS/NGT (49.3\%), 26 (18.5\%) had CS/prediabetes and 43 (30.8\%) had CS/DM. Significant increasing trends in the prevalence of family history of diabetes $(P<0.001)$, metabolic syndrome $(P<0.001)$, age $(P<0.001)$ and waist circumference $(P=0.043)$ and decreasing trends in HOMA- $\beta(P<0.001)$ and oral disposition index (Dlo) $(P<0.002)$ were observed among the groups. No significant trends in fasting insulin levels, area under the curve for insulin ( $\left.A \cup C_{I N S}\right)$, Matsuda index of insulin sensitivity (ISI-Matsuda) and visceral adiposity index were detected.

Conclusions: Impairment of glucose tolerance is characterized by the inability of $\beta$-cells to adequately compensate for insulin resistance through increased insulin secretion. Age, genetic predisposition and lifestyle, in combination with the duration and degree of hypercortisolism, strongly contribute to the impairment of glucose tolerance in patients with a natural history of CS. A careful phenotypic evaluation of glucose tolerance defects in patients with CS proves useful for the identification of those at a high risk of metabolic complications.
\end{abstract}

\section{Introduction}

Cushing's syndrome (CS) results from chronic exposure to glucocorticoid (GC) excess and is characterized by high mortality, mainly due to cardiovascular complications, such as myocardial failure, cerebrovascular disease and thromboembolism $(1,2)$. In a recent cohort study, Dekkers et al. (3) have shown that CS patients with a long-term increased risk of myocardial infarction require adequate monitoring and single-risk factor management.

Diabetes mellitus (DM) is one of the most frequent complications of CS, and its prevalence is considered to range from 20 to $50 \%$, although it may actually be underestimated (4). Indeed, the prevalence of glucose tolerance defects increases up to $70 \%$ when impaired fasting glycaemia (IFG) and impaired glucose tolerance (IGT) are included (5).

Nevertheless, the American Diabetes Association (ADA) continues to define DM in CS as a 'specific type of diabetes secondary to endocrinopathy', although some authors judge it as a classical form of type 2 diabetes $(6,7)$. Indeed, DM in CS occurs as a consequence of insulin (c) 2014 European Society of Endocrinology Printed in Great Britain
Published by Bioscientifica Ltd. 
resistance and impaired insulin secretion induced by GC excess (8). Insulin resistance is due to the effects of GCs on the liver, skeletal muscle and adipose tissue. In particular, in the skeletal muscle, GC excess has major effects on glucose metabolism via interference at several steps in the insulin signalling cascade, resulting in reduced glucose uptake and glycogen synthesis, and on protein metabolism, resulting in proteolysis $(7,8)$. GC excess also leads to increased lipolysis. The consequent elevation of amino acid and free fatty acid levels contributes to further impairment of insulin signalling and favours hepatic gluconeogenesis (4). Moreover, GC excess induces a significant redistribution of adipose tissue from the subcutaneous to the visceral region and also impairment of secretion of adipocytokines such as TNF $\alpha$ (TNF) and IL6 and a decrease in adiponectin levels with an increase in leptin levels $(9,10,11,12)$. Furthermore, it determines $\beta$-cell dysfunction because of reduced insulin sensitivity and direct $\beta$-cell function impairment, through genomic actions that lead to decreased insulin exocytosis and $\beta$-cell apoptosis, which may be further negatively influenced by the treatment undergone $(8,9,10,11,12,13,14)$.

This study aimed to define the changes in insulin secretion and/or sensitivity in a large population of newly diagnosed CS patients according to glucose tolerance and to evaluate whether these changes are influenced by hormonal, metabolic and clinical parameters.

\section{Subjects and methods}

\section{Patients}

We retrospectively revised data collected from 140 consecutive patients with active CS (pituitary and adrenal dependent), newly diagnosed, and recruited at the Endocrinology Units of the Universities of Palermo and Naples between 2000 and 2012.

Data were collected for 113 women (80 with pituitary disease and 33 with adrenal disease, aged $41.67 \pm 15.7$ years) and 27 men (19 with pituitary disease and eight with adrenal disease, aged $38.07 \pm 20.01$ years).

The diagnosis of CS was based, according to international criteria, on high daily urinary free cortisol (UFC) levels (at least three samples), absent cortisol suppression after a low-dose dexamethasone test $(>1.8 \mu \mathrm{g} / \mathrm{dl}$ ) and lack of cortisol rhythm (midnight cortisol levels $>7.5 \mu \mathrm{g} / \mathrm{dl}$ ); in addition, the diagnosis of Cushing's disease was based on the presence of normal or high plasma adrenocorticotrophic hormone (ACTH) levels and adequate cortisol suppression after a high-dose dexamethasone test
( $>80 \%$ ), evidence of pituitary adenoma at RMN and results of inferior petrosal sinus sampling, when required (15). Instrumental examinations were carried out for diagnosis definition.

Exclusion criteria were subclinical CS, adrenal or pituitary cancer, and recurrent CS. No women taking oestrogens and/or progesterone as contraceptives or postmenopausal hormones in replacement therapy were included.

Seven CS patients with IFG were treated with only diet and one with metformin; 13 patients with IGT were treated with diet and four with metformin; one patient with IGF + IGT was treated with metformin; and 12 diabetic patients were treated with diet, 18 with metformin, and one with sulphonylurea. To avoid an effect on insulin sensitivity and secretion indexes, all the diabetic patients treated with oral hypoglycaemic agents stopped taking them 2 days before the evaluation, while 12 patients treated with insulin stopped using bedtime insulin the day before the metabolic evaluation.

This study was approved by the Institutional Review Boards at the Faculty of Medicine of the University of Palermo and Naples. At the time of hospitalization, all the patients signed informed consent for the scientific use of their data. The participants remained anonymous during the database analysis.

\section{Study design}

This is a retrospective, cross-sectional study. All the patients underwent complete clinical and metabolic evaluation as good clinical practice during diagnosis. The patients were subdivided into three groups: normal glucose tolerance (NGT), prediabetes (with IFG, IGT or both) and diabetes (known or newly diagnosed by oral glucose tolerance test (OGTT)). BMI, systolic and diastolic blood pressure, measured according to international criteria (16), waist circumference (WC), measured at the midpoint between the lower rib and the iliac crest, and waist/hip ratio (WHR) were evaluated. After an overnight fast, lipid (total cholesterol, HDL-C and LDL-C, and triglycerides), HbA1c, glycaemia and serum insulin profiles were evaluated.

A complete evaluation of the pituitary-adrenal axis, 8-, 16- and 24-h ACTH and cortisol levels, UFC levels as a mean of three samples from 24-h urine collection, and serum cortisol levels after a low-dose dexamethasone suppression test ( $1 \mathrm{mg}$ ) was carried out. OGTT, performed by measuring plasma blood glucose and insulin levels every $30 \mathrm{~min}$ for $2 \mathrm{~h}$ after a 75-g oral glucose load, was carried out in 66 patients. 
Insulin sensitivity was estimated indirectly using basal insulin and glucose values to calculate the homeostatic model of insulin resistance (HOMA2-IR) (glycaemia $(\mathrm{mmol} / \mathrm{l}) \times$ insulinaemia $(\mu \mathrm{U} / \mathrm{ml}) / 22.5)$ and using glucose and insulin values obtained during the OGTT to calculate the Matsuda index of insulin sensitivity (ISI-Matsuda) $(10000 /$ glucose $\quad(\mathrm{mg} / \mathrm{dl}) \times$ insulin $\quad(\mu \mathrm{U} / \mathrm{ml}) \times$ glucose mean $\times$ insulin mean) $(17,18)$. A composite measure of $\beta$-cell function relative to insulin sensitivity, assessed by the oral disposition index (DIo), was calculated as $\left(\Delta\right.$ Insulin $_{0-30} / \Delta$ Glucose $\left._{0-30}\right) \times(1 /$ fasting insulin). The trapezoidal method was used for the calculation of the area under the curve for insulin ( $\mathrm{AUC}_{2-\mathrm{h} \text { insulin }}$ ) and area under the curve for glucose ( $\mathrm{AUC}_{2 \text {-h glucose }}$ ) (19). The HOMA- $\beta$ was calculated as $360 \times($ insulin $) /($ glucose $)-63) \%$ (glucose in $\mathrm{mg} / \mathrm{dl})(17)$.

The visceral adiposity index (VAI) was calculated according to gender, where TG is the triglyceride level expressed in $\mathrm{mmol} / \mathrm{l}$ and HDL is the HDL-C level expressed in $\mathrm{mmol} / \mathrm{l}$ :

i) $\mathrm{VAI}=(\mathrm{WC} / 39.68+(1.88 \times \mathrm{BMI})) \times(\mathrm{TG} / 1.03) \times$ $(1.31 / \mathrm{HDL})$ for males;

ii) $\mathrm{VAI}=(\mathrm{WC} / 36.58+(1.89 \times \mathrm{BMI})) \times(\mathrm{TG} / 0.81) \times$ (1.52/HDL) (20) for females.

The metabolic syndrome (MS) was diagnosed according to the NCEP ATP III criteria, whereas DM and prediabetes were diagnosed according to the ADA criteria $(6,21)$.

\section{Hormone and biochemical assays}

Serum insulin, glucose and lipid levels were measured by ELISA (DRG Instruments GmbH, Marburg, Germany). The normal insulin range was 5-19 UI/ml. LDL-C levels were measured using the Friedewald formula (total cholesterol$(\mathrm{HDL}+(\mathrm{TG} / 5))$. HbA1c levels were determined by HPLC with an ion-exchange resin (HA8121, Hi-AutoA1c, Menarini, Florence, Italy). UFC levels were measured by RIA (Diagnostic System Laboratories, Inc., Roche Diagnostics $\mathrm{GmbH}$, Mannheim , Germany) with a normal range of $36-137 \mu \mathrm{g} / 24 \mathrm{~h}$. ACTH levels were measured using ELISA (DRG Instruments $\mathrm{GmBH}$ ) with a normal range of 7.6-66.1 pg/ml. Serum cortisol levels were measured using RIA (Diagnostic Systems Laboratories, Inc.) with a reference range of $22.8-217 \mathrm{ng} / \mathrm{ml}$. The conversion factors for the International System (SI) were as follows: glucose $\mathrm{mg} / \mathrm{dl}$ vs $\mathrm{mmol} / \mathrm{l}: 0.0555$; insulin $\mathrm{mUI} / \mathrm{ml}$ vs $\mathrm{pmol} / \mathrm{l}$ : 6.945; total cholesterol and HDL-C mg/dl vs mmol/l: 0.0259; triglycerides mg/dl vs mmol/l: 0.0113; and cortisol ng/ml vs nmol/l: 3.180 .

\section{Statistical analysis}

The Statistical Packages for Social Science SPSS version 17 (SPSS, Inc.) was used for data analysis. The normality of quantitative variables was tested with the Shapiro-Wilk test. Baseline characteristics are presented as means \pm s.D. for continuous variables; rates and proportions were calculated for categorical data. Differences between two groups were detected using unpaired Student's $t$-test for continuous variables (after testing for equality of variance: Levene's test) and the $\chi^{2}$ test and Fisher's exact test (when appropriate) for categorical variables. The ANOVA trend analysis for quantitative variables and $\chi^{2}$ for trend for categorical variables were carried out for the three groups: CS/NGT, CS/prediabetes and CS/DM. A $P$ value $<0.05$ was considered to be statistically significant.

\section{Results}

Metabolic and clinical parameters according to gender

The clinical, phenotypic and biochemical features of all the patients grouped according to gender are listed out in Tables 1, 2 and 3. No differences were found between men and women, except for BMI ( $F$ vs M: $31.35 \pm 6.03$ vs $\left.28.78 \pm 5.52 \mathrm{~kg} / \mathrm{m}^{2} ; P=0.039\right)$ and VAI (F vs M: $2.82 \pm 1.51$ vs $1.46 \pm 0.75 ; P<0.001)$.

\section{Metabolic and clinical parameters according to glucose tolerance}

Of the 140 patients with CS, 71 were classified as having CS/NGT (49.3\%), 26 (18.5\%) CS/prediabetes and 43 CS/DM (30.8\%) (Table 4). Significant increasing trends were observed in age $(\mathrm{CS} / \mathrm{NGT}=35.05 \pm 16.34$; $\mathrm{CS} /$ prediabetes $=41.27 \pm 12.97 ; \mathrm{CS} / \mathrm{DM}=50.58 \pm 14.64$ years; $P<0.001)$ and $\mathrm{WC}(\mathrm{CS} / \mathrm{NGT}=100.85 \pm 14.77 ; \mathrm{CS} /$ prediabetes $=$ $106.28 \pm 17.49 ; \mathrm{CS} / \mathrm{DM}=106.76 \pm 16.00 \mathrm{~cm} ; P=0.043)$. A non-significant trend was observed in BMI (CS/NGT $=$ $30.00 \pm 5.07 ; \quad \mathrm{CS} /$ prediabetes $=31.18 \mathrm{~kg} / \mathrm{m}^{2} \quad \pm 6.08$; $\mathrm{CS} / \mathrm{DM}=32.07 \pm 7.19 ; P=0.072$ ) (Table 4 ).

In addition, an increasing trend was observed in the familial history of DM $(\mathrm{CS} / \mathrm{NGT}=29.6 \%$; $\mathrm{CS} /$ prediabetes $=$ $46.2 \% ; \mathrm{CS} / \mathrm{DM}=65.1 \% ; P<0.001)$ and cardiovascular disease $\quad(\mathrm{CS} / \mathrm{NGT}=54.9 \% ; \quad \mathrm{CS} /$ prediabetes $=76.9 \%$; $\mathrm{CS} / \mathrm{DM}=81.4 \% ; \quad P=0.002)$, MS $(\mathrm{CS} / \mathrm{NGT}=39.4 \% ; \mathrm{CS} /$ prediabetes $=76.9 \% ; \quad \mathrm{CS} / \mathrm{DM}=93 \% ; \quad P<0.001) \quad$ and hypertension $(\mathrm{CS} / \mathrm{NGT}=67.6 \%$; $\mathrm{CS} /$ prediabetes $=76.9 \%$; $\mathrm{CS} / \mathrm{DM}=86 \% ; P<0.026$ ) (Table 4 ). 
Table 1 Clinical and phenotypic features of all the 140 CS patients at diagnosis grouped according to gender. Data is presented as mean \pm s.D. or as $n(\%)$.

\begin{tabular}{l}
\hline \\
\hline Age (years) \\
BMI $\left(\mathrm{kg} / \mathrm{m}^{2}\right)$ \\
WC \\
WHR \\
Family history of \\
Diabetes \\
Cardiovascular disease \\
Dyslipidaemia \\
Smoking \\
Pituitary disease \\
Adrenal disease \\
Autoimmune thyroid disease \\
Multinodular goitre \\
Muscle hypotrophy \\
Moon face \\
Facial plethora \\
Buffalo hump \\
Purple striae \\
Ecchymosis \\
Coronary heart disease \\
Coagulopathy \\
Peripheral vascular disease \\
Cerebral vascular disease \\
Depression \\
Osteoporosis \\
Fractures \\
Vertebral collapse \\
Arthropathy \\
Hepatic steatosis \\
\hline
\end{tabular}

\begin{tabular}{c}
\hline Women $(n=113)$ \\
\hline $41.67 \pm 15.7$ \\
$31.35 \pm 6.03$ \\
$104.04 \pm 15.53$ \\
$1.01 \pm 0.09$ \\
\\
$48(42.5)$ \\
$75(66.4)$ \\
$30(26.5)$ \\
$40(35.4)$ \\
$80(78.8)$ \\
$33(29.2)$ \\
$13(11.5)$ \\
$14(12.4)$ \\
$46(40.7)$ \\
$58(51.3)$ \\
$59(52.2)$ \\
$44(38.9)$ \\
$45(39.8)$ \\
$25(22.1)$ \\
$19(16.8)$ \\
$7(6.2)$ \\
$5(4.4)$ \\
$10(8.8)$ \\
$27(23.9)$ \\
$25(22.1)$ \\
$10(8.8)$ \\
$11(9.7)$ \\
$39(34.5)$ \\
$42(57.5)$ \\
\end{tabular}

\begin{tabular}{c}
\hline Men $(n=27)$ \\
\hline $38.07 \pm 20.01$ \\
$28.78 \pm 5.52$ \\
$102.16 \pm 17.33$ \\
$1.03 \pm 0.05$
\end{tabular}

\begin{tabular}{crr}
\hline Total $(n=140)$ & & $\boldsymbol{P}$ \\
\cline { 1 - 1 } $40.98 \pm 16.61$ & & 0.314 \\
$30.86 \pm 6.01$ & & 0.039 \\
$103.68 \pm 15.83$ & & 0.609 \\
$1.01 \pm 0.09$ & & 0.242
\end{tabular}

13 (48.1)

19 (70.4)

9 (33.3)

14 (51.9)

19 (70.4)

8 (29.6)

$1(0.4)$

2 (7.4)

9 (33.3)

12 (44.4)

$13(48.1)$

$6(22.2)$

13 (48.1)

7 (25.9)

4 (14.8)

$3(11.1)$

2 (7.4)

$2(7.4)$

5 (18.5)

7 (25.9)

5 (18.5)

$2(7.4)$

8 (29.6)

$9(69.2)$

$61(43.6)$
$94(74.1)$
$39(27.9)$
$54(38.6)$
$99(70.7)$
$41(29.3)$
$14(10)$
$16(11.4)$
$55(39.3)$
$70(50)$
$72(51.4)$
$50(35.7)$
$58(41.4)$
$32(22.9)$
$23(16.4)$
$10(7.2)$
$7(5)$
$12(8.6)$
$32(22.9)$
$32(22.9)$
$15(10.7)$
$13(9.3)$
$47(33.6)$
$51(59.3)$

0.593

0.691

0.480

0.115

0.965

0.965

0.607

0.737

0.481

0.520

0.704

0.103

0.430

0.673

1

0.406

0.620

1

0.550

0.673

0.144

1

0.629

0.547

No differences were found in venous thrombosis, fasting insulin levels, total HDL-C and LDL-C levels, triglyceride

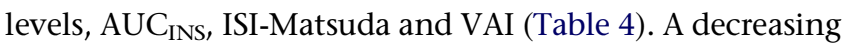
trend was observed in HOMA- $\beta(\mathrm{CS} / \mathrm{NGT}=170.65 \pm 43.07$; $\mathrm{CS} /$ prediabetes $=163.46 \pm 77.52 ; \mathrm{CS} / \mathrm{DM}=87.11 \pm 76.94 ;$ $P<0.001)$ and DIo $(\mathrm{CS} / \mathrm{NGT}=3.04 \pm 3.68$; CS/prediabetes $=0.87 \pm 1.12 ; \mathrm{CS} / \mathrm{DM}=0.70 \pm 1.07 ; P<0.001)$ (Table 4$)$. As expected, a significant increasing trend was observed in fasting glucose levels $(\mathrm{CS} / \mathrm{NGT}=4.77 \pm 0.64 ; \mathrm{CS} /$ prediabetes $=5.43 \pm 0.97 ; \quad \mathrm{CS} / \mathrm{DM}=7.79 \pm 2.68 \mathrm{mmol} / \mathrm{l}$; $P<0.001)$, AUC $_{2-\mathrm{h}}$ glucose $(\mathrm{CS} / \mathrm{NGT}=14756 \pm 3038 ; \mathrm{CS} /$ prediabetes $=17314 \pm 4890 ; \quad \mathrm{CS} / \mathrm{DM}=24707 \pm 8882$; $P=0.005)$ and HbA1c levels (CS/NGT $=34.97 \pm 3.4 ; \mathrm{CS} /$ prediabetes $=39.89 \pm 5.1 ; \mathrm{CS} / \mathrm{DM}=54.20 \pm 12.4 \mathrm{mmol} / \mathrm{mol}$; $P<0.001$ ) (Table 4).

\section{Hormonal parameters according to glucose tolerance}

Circadian rhythms for ACTH and cortisol, UFC and cortisol after the administration of $1 \mathrm{mg}$ dexamethasone did not differ significantly among the three groups (Table 4).

\section{Discussion}

The results of this study indicate that, in agreement with the literature data, the prevalence of CS is high in women $(80.71 \%)$. The sexual dimorphism of CS in women has already been documented (22). To date, it is estimated that Cushing's disease occurs five times more frequently in women than in men (23). Women with CS have a higher BMI than men, with similar values of WC, because in CS the gender differences in fat distribution are lost precisely because the adipose tissue is redistributed from the peripheral to the visceral region, leading to the wellknown phenotype characterized by gynoid obesity, independently of sex $(24,25,26,27)$. In our study, CS women had a significantly higher VAI, consequent on the influence of GC excess on visceral adipose dysfunction. The VAI is a gender-specific index, which was modelled on healthy men and women $(20,28)$. The increase in the VAI, specifically observed in CS women, confirms the loss of cardiovascular protection in relation to gender, and CS women even developed worse visceral adipose dysfunction (indirectly expressed by the VAI) compared with men, 
Table 2 Hormonal parameters of all the 140 CS patients at diagnosis grouped according to gender. Data is presented as mean \pm s.D.

\begin{tabular}{l}
\hline \\
\hline 8-h ACTH $(\mathrm{pg} / \mathrm{ml})$ \\
$16-\mathrm{h}$ ACTH $(\mathrm{pg} / \mathrm{ml})$ \\
24-h ACTH $(\mathrm{pg} / \mathrm{ml})$ \\
8-h Cortisol $(\mathrm{nmol} / \mathrm{l})$ \\
16-h Cortisol $(\mathrm{nmol} / \mathrm{l})$ \\
24-h Cortisol $(\mathrm{nmol} / \mathrm{l})$ \\
Urinary free cortisol $(\mu / 24 \mathrm{~h})$ \\
Cortisol after an overnight \\
$\quad$ dexamethasone suppression \\
$\quad$ test (nmol/l)
\end{tabular}

\begin{tabular}{c}
\hline Women $(n=113)$ \\
\hline $55.13 \pm 44.27$ \\
$45.48 \pm 36.2$ \\
$48.14 \pm 27.42$ \\
$817.26 \pm 225.78$ \\
$747.30 \pm 232.14$ \\
$629.64 \pm 313.73$ \\
$393.39 \pm 348.45$ \\
$295.74 \pm 248.04$ \\
\end{tabular}

\begin{tabular}{c}
\hline Men $(n=27)$ \\
\hline $58.79 \pm 46.89$ \\
$44.33 \pm 34.12$ \\
$41.76 \pm 35.2$ \\
$791.82 \pm 279.8$ \\
$610.56 \pm 241.68$ \\
$508.8 \pm 225.78$ \\
$518.92 \pm 457.98$ \\
$397.5 \pm 384.78$
\end{tabular}

\begin{tabular}{ccc} 
Total $(n=140)$ & & $\boldsymbol{P}$ \\
\cline { 1 - 1 } $55.85 \pm 44.64$ & & 0.715 \\
$45.42 \pm 35.69$ & & 0.969 \\
$46.26 \pm 29.79$ & & 0.475 \\
$814.08 \pm 235.32$ & & 0.626 \\
$728.22 \pm 238.5$ & & 0.142 \\
$594.66 \pm 292.56$ & & 0.115 \\
$416.87 \pm 372.76$ & & 0.122 \\
$314.82 \pm 276.66$ & & 0.344
\end{tabular}

as also observed in other endocrine diseases, such as acromegaly, prolactinoma and diabetes $(28,29,30,31)$. However, despite this evidence, the VAI in CS has a strong application limit. The reason for this is that it is an indicator of early cardiometabolic risk and an important diagnostic tool in all borderline conditions in which overt MS is not present (32). Indeed, in our cohort of CS patients, $62.9 \%$ had overt MS, and so the VAI cannot be considered a specific index for these patients. This is explained by the fact that three of the variables making up the VAI (WC, TG and HDL) are dichotomically expressed in the criteria of MS. In a recent letter, the applicative limits of VAI in single patients have been pointed out and it has been recommended that for proper application in an individual patient or in small sample studies the application of the VAI is not suggested, above all in the

Table 3 Metabolic parameters of all the 140 CS patients at diagnosis grouped according to gender. Data is presented as $n(\%)$ or as mean \pm s.D.

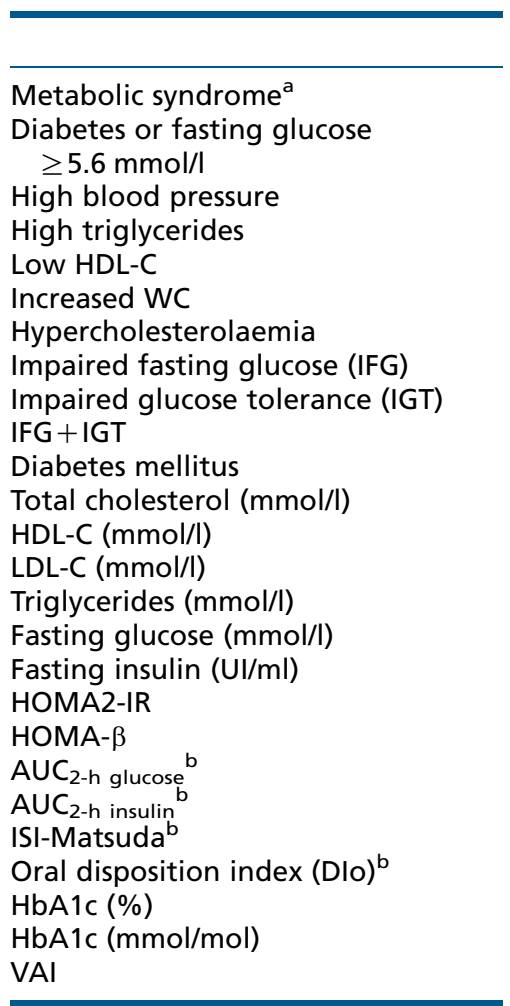

\begin{tabular}{c} 
Women $(n=113)$ \\
\hline $75(66.4)$ \\
$42(37)$ \\
$84(74.3)$ \\
$38(33.6)$ \\
$72(63.7)$ \\
$94(83.2)$ \\
$49(43.4)$ \\
$6(5.3)$ \\
$14(12.4)$ \\
0 \\
$36(31.9)$ \\
$5.19 \pm 0.93$ \\
$1.27 \pm 0.33$ \\
$3.11 \pm 0.87$ \\
$1.58 \pm 0.60$ \\
$5.79 \pm 2.08$ \\
$13.16 \pm 6.28$ \\
$2.00 \pm 0.95$ \\
$142.99 \pm 69.9$ \\
$18168 \pm 7180$ \\
$9627 \pm 5721$ \\
$4.16 \pm 3.48$ \\
$1.88 \pm 3.07$ \\
$6.24 \pm 1.13$ \\
$45.0 \pm 12.4$ \\
$2.76 \pm 1.41$ \\
\end{tabular}

\begin{tabular}{c}
\hline Men $(n=27)$ \\
\hline $13(48.1)$ \\
$10(8)$
\end{tabular}

\begin{tabular}{c}
\hline Total $(n=140)$ \\
\hline $88(62.9)$ \\
$52(46)$
\end{tabular}

\begin{tabular}{c}
\hline $\boldsymbol{P}$ \\
\hline 0.078 \\
0.017
\end{tabular}

High blood pressure

$21(67.8)$

$9(33.3)$

$5(18.5)$

$20(74.1)$

$12(44.4)$

2 (7.4)

3 (11.1)

1 (3.7)

7 (25.9)

$4.79 \pm 0.88$

$1.35 \pm 0.36$

$2.74 \pm 0.79$

$1.32 \pm 0.57$

$5.97 \pm 2.12$

$14.73 \pm 7.53$

$2.21 \pm 1.10$

$146.44 \pm 88.13$

$17822 \pm 5703$

$9602 \pm 6696$

$3.53 \pm 1.83$

$1.43 \pm 1.27$

$6.5 \pm 1.13$

$48.0 \pm 12.4$

$1.43 \pm 1.27$

$105(75)$
$47(33.6)$
$77(55)$
$114(81.4)$
$61(43.6)$
$8(5.7)$
$17(12.1)$
$1(0.7)$
$43(30.7)$
$5.11 \pm 0.93$
$1.29 \pm 0.34$
$3.04 \pm 0.86$
$1.53 \pm 0.60$
$5.82 \pm 2.08$
$13.46 \pm 6.54$
$2.04 \pm 0.98$
$143.66 \pm 72.38$
$18095 \pm 6854$
$9622 \pm 5891$
$4.02 \pm 3.19$
$1.78 \pm 2.78$
$6.29 \pm 1.13$
$45.0 \pm 12.4$
$2.51 \pm 1.40$

0.711

0.977

$<0.001$

0.279

0.919

0.651

1

0.193

0.548

0.049

0.243

0.037

0.059

0.753

0.407

0.432

0.935

0.987

0.974

0.615

0.913

0.357

0.357

$<0.001$

${ }^{a}$ According to the Adult Treatment Panel (ATP) III criteria. 
Table 4 Clinical and biochemical features of all the $140 \mathrm{CS}$ patients divided into three groups (normal glucose tolerance (CS/NGT), prediabetes (CS/prediabetes) and diabetes (CS/DM)). Data is presented as mean \pm s.D. or as $n(\%)$.

\begin{tabular}{l}
\hline \\
Age (years) \\
BMI (kg/m²) \\
WC \\
WHR \\
Male \\
Female \\
Family history of \\
Diabetes \\
Cardiovascular disease \\
Dyslipidaemia \\
Smoking \\
Pituitary disease \\
Adrenal disease \\
Metabolic syndrome \\
Hypertension \\
Venous thrombosis \\
Hormonal parameters \\
8-h ACTH (pg/ml) \\
16-h ACTH (pg/ml) \\
24-h ACTH (pg/ml) \\
8-h Cortisol (nmol/l) \\
16-h Cortisol (nmol/l) \\
24-h Cortisol (nmol/l) \\
Urinary free cortisol ( $\mu / 24$ h) \\
Cortisol after an overnight \\
dexamethasone suppression \\
test (nmol/l) \\
Metabolic parameters \\
Total cholesterol (mmol/l) \\
HDL-C (mmol/l) \\
LDL-C (mmol/l) \\
Triglycerides (mmol/l) \\
Fasting glucose (mmol/l) \\
Fasting insulin (Ul/ml) \\
HOMA2-IR \\
HOMA- $\beta$ \\
AUC2-h glucose \\
AUC $2-\mathrm{h}$ insulin \\
ISI-Matsudab \\
Oral disposition index (Dlo) \\
HbA1c (\%) \\
HbA1c (mmol/mol) \\
VAl \\
\hline
\end{tabular}

\begin{tabular}{c}
\hline CS/NGT $(n=71)$ \\
\hline $35.05 \pm 16.34$ \\
$30.00 \pm 5.07$ \\
$100.85 \pm 14.77$ \\
$1.01 \pm 0.09$ \\
$14(19.7)$ \\
$57(80.3)$ \\
$21(29.6)$ \\
$39(54.9)$ \\
$18(25.4)$ \\
$23(32.4)$ \\
$48(67.6)$ \\
$23(32.4)$ \\
$28(39.4)$ \\
$48(67.6)$ \\
$7(5)$
\end{tabular}

\begin{tabular}{c}
\hline CS/prediabetes $(n=26)$ \\
\hline $41.27 \pm 12.97$ \\
$31.18 \pm 6.08$ \\
$106.28 \pm 17.49$ \\
$1.02 \pm 0.09$ \\
$6(23.1)$ \\
$20(76.9)$ \\
$12(46.2)$ \\
$20(76.9)$ \\
$10(38.5)$ \\
$10(38.5)$ \\
$21(80.8)$ \\
$5(19.2)$ \\
$20(76.9)$ \\
$20(76.9)$ \\
$5(4.4)$
\end{tabular}

\begin{tabular}{c}
\hline CS/DM $(n=43)$ \\
\hline $50.58 \pm 14.64$ \\
$32.07 \pm 7.19$ \\
$106.76 \pm 16.00$ \\
$1.01 \pm 0.08$ \\
$7(16.3)$ \\
$36(83.7)$ \\
$28(65.1)$ \\
$35(81.4)$ \\
$11(25.6)$ \\
$21(48.8)$ \\
$30(69.8)$ \\
$13(30.2)$ \\
$40(93)$ \\
$37(86)$ \\
$2(7.4)$
\end{tabular}

\begin{tabular}{r}
\multicolumn{1}{c}{$\boldsymbol{P}$} \\
$<0.001$ \\
0.072 \\
0.043 \\
0.900 \\
0.696 \\
0.696 \\
$<0.001$ \\
0.002 \\
0.863 \\
0.082 \\
0.704 \\
0.704 \\
$<0.001$ \\
0.026 \\
0.620
\end{tabular}

$51.11 \pm 42.87$

$40.90 \pm 36.18$

$41.27 \pm 31.00$

$782.28 \pm 209.88$

$734.58 \pm 190.8$

$556.5 \pm 238.5$

$436.25 \pm 334.20$

$302.1 \pm 273.48$

$65.85 \pm 34.47$

$55.58+35.24$

$52.66 \pm 20.32$

$817.26 \pm 235.32$

$721.86 \pm 228.96$

$607.38 \pm 295.74$

$376.82 \pm 322.03$

$333.9 \pm 263.94$

$4.95 \pm 0.87$
$1.31 \pm 0.35$
$2.94 \pm 0.83$
$1.47 \pm 0.71$
$4.77 \pm 0.64$
$13.54 \pm 5.23$
$1.97 \pm 0.79$
$170.65 \pm 43.07$
$14756 \pm 3038$
$10615 \pm 6259$
$4.25 \pm 1.93$
$3.04 \pm 3.68$
$5.35 \pm 0.31$
$34.97 \pm 3.4$
$2.36 \pm 1.44$

$5.60 \pm 1.08$

$1.32 \pm 0.37$

$3.36 \pm 0.99$

$1.74 \pm 0.97$

$5.43 \pm 0.97$

$17.06 \pm 8.05$

$2.57 \pm 1.19$

$163.46 \pm 77.52$

$17314 \pm 4890$

$10398 \pm 5769$

$3.35 \pm 1.95$

$0.87 \pm 1.12$

$5.80 \pm 0.47$

$39.89 \pm 5.1$

$2.43 \pm 1.11$

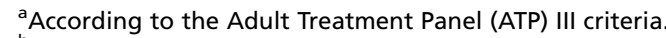

${ }^{b}$ In a subgroup of 66 patients without known diabetes.

$\begin{array}{ll}57.86 \pm 52.00 & 0.365 \\ 45.58 \pm 35.47 & 0.530 \\ 51.00 \pm 31.50 & 0.209 \\ 858.6 \pm 270.3 & 0.102 \\ 718.68 \pm 340.26 & 0.815 \\ 645.54 \pm 365.7 & 0.249 \\ 409.54 \pm 457.89 & 0.668 \\ 321.18 \pm 292.56 & 0.783\end{array}$

presence of morbid obesity, pendulous abdomen, severe hypertriglyceridaemia and/or fibrate use (33).

The results of our study indicate that DM patients are significantly older, have a higher prevalence of family history of DM and exhibit a marked deterioration of insulin secretion indexes.

The familial history of DM appears to predispose CS patients to worse metabolic derangement. It is worth noting that DM is a polygenic disease, and among all the genetic factors described, the presence of relatives affected by the disease remains one of the most important determinants for predicting the onset of disease (34). The role of age in the development of type 2 diabetes is unanimously recognized, and these findings were confirmed in our cohort, i.e. CS/DM patients are older than CS/NGT and CS/prediabetes patients.

A condition of insulin resistance has been reported in hypercortisolism (35). Surprisingly, neither ISI-Matsuda nor HOMA-IR was significantly affected in CS/DM patients in comparison with that in the other CS patients. By 
contrast, a worsening of insulin secretion relative to insulin resistance (DIo), but not of absolute insulin secretion $\left(\mathrm{AUC}_{\mathrm{INS}}\right.$ ), was observed in $\mathrm{CS} / \mathrm{DM}$ patients in addition to the reduction of HOMA- $\beta(3,12)$. Indeed, the DIo, which expresses the ability of $\beta$-cells to adequately compensate for insulin resistance through increased insulin secretion, has been shown to predict the development of diabetes in adults (19). Therefore, the DIo could be used for evaluating diabetes risk linked to the CS condition.

These data show that patients with CS/DM have a strong insulin secretion defect without a worsening of insulin sensitivity in comparison with CS/NGT and CS/prediabetes patients. Therefore, diabetes in CS could probably be strongly influenced by $\beta$-cell defect that would occur after a long period of insulin resistance. This observation is not new, even if the data available are on mice or on patients with exogenous hypercortisolism (36). However, these data need to be investigated further.

Recently, many studies have been conducted on the usefulness of evaluating the hypothalamus-pituitaryadrenal axis in patients with DM. To date, this evaluation is not recommended $(37,38)$. Moreover, there are no data that can define any differences between patients with classic DM and those with CS and DM.

From the data obtained in our study, we can hypothesize that a time-dependent prodromal period, influencing both CS and DM, progresses together with other variables that can play a distinct role in the determination of diabetes in CS, such as the duration of disease, degree of hypercortisolism and genetic factors. The first consideration points out that the duration of disease is a parameter that is not always determinable in CS because the onset of CS is subtle and is usually diagnosed some time after the real onset of the disease. On the other hand, the degree of hypercortisolism may be variable: obviously marked hypercortisolism should exert an effect different from that of slight hypercortisolism. In this light, recently, it has been suggested that GC action may be influenced by GC receptor (GR (NR3C1)) polymorphisms (39). Some $G R$ polymorphisms have been demonstrated to impair GC sensitivity and can be associated with altered metabolic profiles. In particular, N363S, BCL1 and ER22/EK are associated with diabetes, hypertension and dyslipidaemia, while A3669G GR polymorphism seems to be protective in CS (39).

A further confirmation of the complex and heterogeneous variables implicated in the development of diabetes in CS is emerging from the different effects of pasireotide on glucose tolerance in patients with CS: some

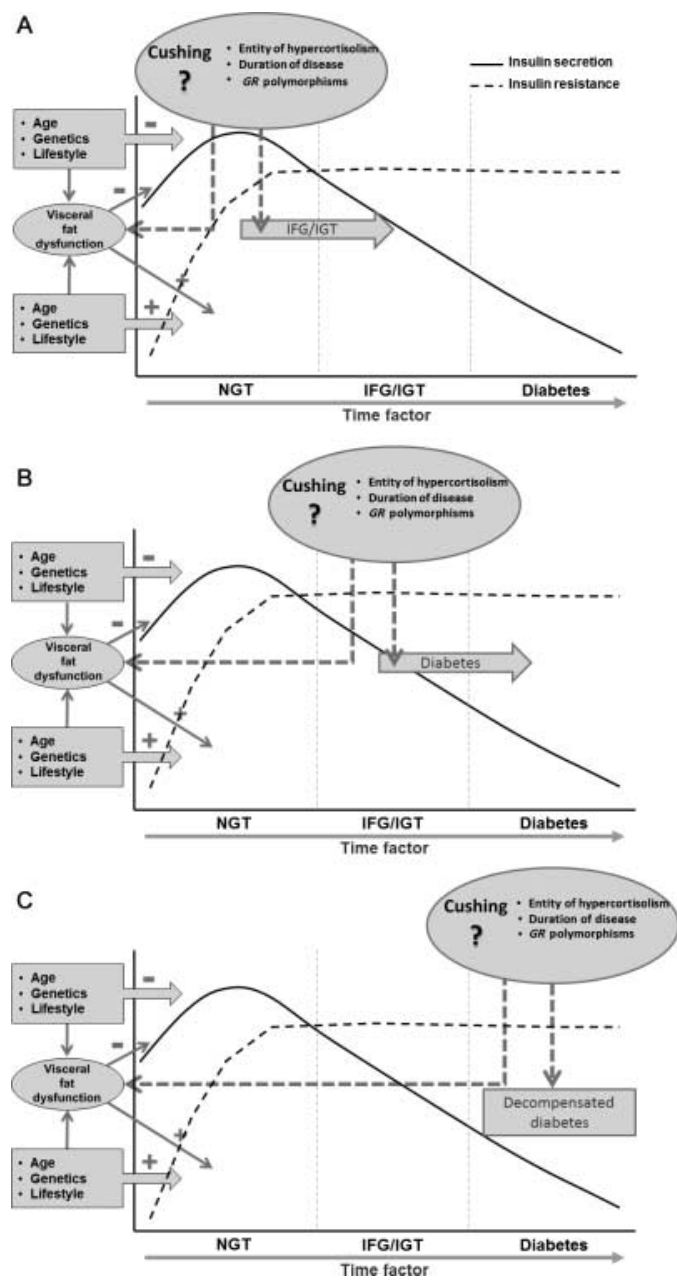

Figure 1

Diabetes in CS is due to complex and heterogeneous pathophysiological mechanisms linked only in part to insulin resistance.

(A) Patients with normal glucose tolerance. Age, genetic predisposition and lifestyle in association with visceral adipose dysfunction determine an early onset of insulin resistance and trigger reduction of insulin secretion. CS onset, through its specific factors, favours the impairment of glucose tolerance and the development of IFG/IGT (latent diabetes is triggered). (B) Patients with IFG/IGT. Age, genetic predisposition and lifestyle in association with visceral adipose dysfunction determine a worsening of insulin resistance and a significant reduction of insulin secretion. CS onset, through its specific factors, favours the impairment of glucose tolerance and the development of diabetes (latent diabetes is revealed). (C) Patients with diabetes. Age, genetic predisposition and lifestyle in association with visceral adipose dysfunction determine a significant progression of insulin resistance and a strong reduction of insulin secretion. CS onset, through its specific factors, together with the DM-specific ones, completely eliminates glucose tolerance, determining the decompensation of diabetes (diabetes mellitus is overt). 
of them can develop overt diabetes, while others maintain NGT. This difference is most probably due to the interplay between pasireotide $\beta$-cell effects and genetic/ environmental factors (40). Moreover, the complexity of the pathogenesis of diabetes in CS could also be supported by the evidence that in a large number of patients with CS, DM persists also after definitive treatment when total resolution of CS has been achieved (41).

Based on the above, we could suggest that in CS diabetes is due to the action of non-modifiable risk factors, such as age and genetic predisposition, and modifiable risk factors, such as lifestyle, which are independent of hypercortisolism. When CS becomes overt through some specific factors, such as the exact duration of hypercortisolism, GR polymorphisms and degree of hypercortisolism, it contributes to the impairment of glucose tolerance. Therefore, in the presence of the abovementioned characteristics, if a patient has NGT, s/he will develop prediabetes or diabetes; if a patient has prediabetes, s/he will develop DM; and if a patient is diabetic, s/he will exhibit a worsening of glycaemic control with decompensated diabetes (Fig. 1).

Clearly, obesity and MS are additional factors that are associated with chronic inflammation contributing to the further development of insulin resistance and progression to diabetes. Local hyperactivity of GCs in abdominal adipose tissue contributes to the pathogenesis of MS and ongoing development of DM, steps that may vary in a CS patient in relation to different mechanisms arising in the history of the disease $(27,42)$. Therefore, careful phenotypic evaluation of glucose tolerance defects in patients with CS is necessary, using, if possible, accurate examinations to verify insulin secretion and sensitivity, before beginning medical treatment for CS, which can further impair glucose tolerance. In addition, this phenotypic evaluation could be essential above all for the identification of the best therapeutic management of diabetes in CS.

In conclusion, the results of this study suggest that DM is not a simple consequence of hypercortisolism and that both genetic predisposition for DM and hypercortisolism, working together, exert dangerous effects on glucose metabolism. However, increased insulin resistance and decreased insulin secretion entities may be extremely different in a single CS patient during the course of the disease.

\section{Declaration of interest}

The authors declare that there is no conflict of interest that could be perceived as prejudicing the impartiality of the research reported.

\section{Funding}

This research did not receive any specific grant from any funding agency in the public, commercial or not-for-profit sector.

\section{References}

1 Pivonello R, De Martino MC, De Leo M, Lombardi G \& Colao A. Cushing's syndrome. Endocrinology and Metabolism Clinics of North America 200837 135-149. (doi:10.1016/j.ecl.2007.10.010)

2 Colao A, Pivonello R, Spiezia S, Faggiano A, Ferone D, Filippella M, Marzullo P, Cerbone G, Siciliani M \& Lombardi G. Persistence of increase cardiovascular risk in patients with Cushing's disease after five years of successful cure. Journal of Clinical Endocrinology and Metabolism 199984 2664-2672. (doi:10.1210/jc.84.8.2664)

3 Dekkers OM, Horváth-Puhó E, Jørgensen JO, Cannegieter SC, Ehrenstein V, Vandenbroucke JP, Pereira AM \& Sørensen HT. Multisystem morbidity and mortality in Cushing's syndrome: a cohort study. Journal of Clinical Endocrinology and Metabolism 201398 2277-2284. (doi:10.1210/jc.2012-3582)

4 Pivonello R, De Leo M, Vitale P, Cozzolino A, Simeoli C, De Martino MC, Lombardi G \& Colao A. Pathophysiology of diabetes mellitus in Cushing's syndrome. Neuroendocrinology 201092 (Suppl 1) 77-81. (doi:10.1159/000314319)

5 Resmini E, Minuto F, Colao A \& Ferone D. Secondary diabetes associated with principal endocrinopathies: the impact of new treatment modalities. Acta Diabetologica 200945 85-95. (doi:10.1007/ s00592-009-0112-9)

6 American Diabetes Association. Diagnosis and classification of diabetes mellitus. Diabetes Care 201336 (Suppl 1) S67-S74.

7 McMahon M, Gerich J \& Rizza R. Effects of glucocorticoids on carbohydrate metabolism. Diabetes/Metabolism Reviews 1988 4 17-30. (doi:10.1002/dmr.5610040105)

8 Van Raalte DH, Ouwens DM \& Diamant M. Novel insights into glucocorticoid-mediated diabetogenic effects: towards expansion of therapeutic options? European Journal of Clinical Investigation 200939 81-93. (doi:10.1111/j.1365-2362.2008.02067.x)

9 Fasshauer M \& Paschke R. Regulation of adipocytokines and insulin resistance. Diabetologia 200346 1594-1603. (doi:10.1007/s00125003-1228-z)

10 Lundgren M, Buren J, Ruge T, Myrnas T \& Eriksson JW. Glucocorticoids down-regulate glucose uptake capacity and insulin-signaling proteins in omental but not subcutaneous human adipocytes. Journal of Clinical Endocrinology and Metabolism 200489 2989-2997. (doi:10.1210/ jc.2003-031157)

11 Valassi E, Biller BM, Klibanski A \& Misra M. Adipokines and cardiovascular risk in Cushing's syndrome. Neuroendocrinology 201295 187-206. (doi:10.1159/000330416)

12 Martin SS, Qasim A \& Reilly MP. Leptin resistance: a possible interface of inflammation and metabolism in obesity-related cardiovascular disease. Journal of the American College of Cardiology 200852 1201-1210. (doi:10.1016/j.jacc.2008.05.060)

13 Mazziotti G, Gazzaruso C \& Giustina A. Diabetes in Cushing's syndrome. Basic and clinical aspects. Trends in Endocrinology and Metabolism 201122 499-506. (doi:10.1016/j.tem.2011.09.001)

14 Van Raalte DH, Mari A, Bunck MC, Nofrate V, Dokter W \& Nassander U. Acute and subacute exposure to glucocorticoids differentially impair various aspects of $\beta$-cell function in healthy males (1468-P). 2008 68th Sessions American Diabetes Association. San Francisco.

15 Nieman LK, Biller BM, Findling JW, Newell-Price J, Savage MO, Stewart PM \& Montori VM. The diagnosis of Cushing's syndrome: an Endocrine Society Clinical Practice Guideline. Journal of Clinical Endocrinology and Metabolism 200893 1526-1540. (doi:10.1210/ jc.2008-0125) 
16 Withworth JA. World Health Organization (WHO)/International Society of Hypertension (ISH) statement on management of hypertension. Journal of Hypertension 200321 1983-1992. (doi:10.1097/ 00004872-200311000-00002)

17 Matthews D, Hosker J, Rudenski A, Naylor BA, Treacher DF \& Turner RC. Homeostasis model assessment: insulin resistance and $\beta$-cell function from fasting plasma glucose and insulin concentration in man. Diabetologia 198528 412-419. (doi:10.1007/BF00280883)

18 Matsuda M \& DeFronzo R. Insulin sensitivity indices obtained from oral glucose tolerance testing: comparison with the euglycemic insulin clamp. Diabetes Care 199922 1462-1470. (doi:10.2337/diacare.22.9.1462)

19 Utzschneider KM, Prigeon RL, Faulenbach MV, Tong J, Carr DB, Boyko EJ, Leonetti DL, McNeely MJ, Fujimoto WY \& Kahn SE. Oral disposition index predicts the development of future diabetes above and beyond fasting and 2-h glucose levels. Diabetes Care 200932 335-341. (doi:10.2337/dc08-1478)

20 Amato MC, Giordano C, Galia M, Criscimanna A, Vitabile S, Midiri M \& Galluzzo A \& AlkaMesy Study Group. Visceral adiposity index: a reliable indicator of visceral fat function associated with cardiometabolic risk. Diabetes Care 201033 920-922. (doi:10.2337/dc09-1825)

21 Expert Panel on Detection, Evaluation and Treatment of High Blood Cholesterol in Adults. Executive summary of the Third Report of the National Cholesterol Education Program (NCEP) Expert Panel on Detection, Evaluation and Treatment of High Blood Cholesterol in Adults (Adult Treatment Panel III). Journal of the American Medical Association 2001285 2486-2497. (doi:10.1001/jama.285.19.2486)

22 Invitti C, Pecori Giraldi F, De Martin M \& Cavagnini F. Diagnosis and management of Cushing's syndrome: results of an Italian multicentre study. Journal of Clinical Endocrinology and Metabolism 1999 84 440-448. (doi:10.1210/jc.84.2.440)

23 Valassi E, Santos A, Yaneva M, Tóth M, Strasburger CJ, Chanson P, Wass JA, Chabre O, Pfeifer M, Feelders RA et al. Baseline demographic and clinical characteristics. European Journal of Endocrinology 2011165 383-392. (doi:10.1530/EJE-11-0272)

24 Rockall AG, Sohaib SA, Evans D, Kaltsas G, Isidori AM, Monson JP, Besser GM, Grossman A \& Reznek RH. Computed tomography assessment of fat distribution in male and female patients with Cushing's syndrome. European Journal of Endocrinology 2003149 561-567. (doi:10.1530/eje.0.1490561)

25 Shibli-Rahhal A, Van Beek M \& Schlechte JA. Cushing's syndrome. Clinics in Dermatology 200624 260-265. (doi:10.1016/j.clindermatol. 2006.04.012)

26 Anagnostis P, Athyros VG, Tziomalos K, Karagiannis A \& Mikhailidis DP. The pathogenic role of cortisol in the metabolic syndrome: a hypothesis. Journal of Clinical Endocrinology and Metabolism 200994 2692-2701. (doi:10.1210/jc.2009-0370)

27 Vegiopoulos A \& Herzig S. Glucorticoids, metabolism and metabolic diseases. Molecular and Cellular Endocrinology 2007275 43-61. (doi:10.1016/j.mce.2007.05.015)

28 Amato MC, Guarnotta V \& Giordano C. Body composition assessment for the definition of cardiometabolic risk. Journal of Endocrinological Investigation 201336 537-543. (doi:10.3275/8943)

29 Amato MC, Giordano C, Pitrone M \& Galluzzo A. Cut-off points of the visceral adiposity index (VAI) identifying a visceral adipose dysfunction associated with cardiometabolic risk in a Caucasian Sicilian population. Lipids in Health and Disease 201110 183. (doi:10.1186/ 1476-511X-10-183)

30 Ciresi A, Amato MC, Pivonello R, Nazzari E, Grasso LF, Minuto F, Ferone D, Colao A \& Giordano C. The metabolic profile in active acromegaly is gender-specific. Journal of Clinical Endocrinology and Metabolism 201398 E51-E59. (doi:10.1210/jc.2012-2896)

31 Ciresi A, Amato MC, Guarnotta V, Lo Castro F \& Giordano C. Higher doses of cabergoline further improve metabolic parameters in patients with prolactinoma regardless of the degree of reduction in prolactin levels. Clinical Endocrinology 201379 845-952. (doi:10.1111/cen.12204)

32 Al-Daghri NM, Al-Attas OS, Alokail MS, Alkharfy KM, Charalampidis P, Livadas S, Kollias A, Sabico SL \& Chrousos GP. Visceral adiposity index is highly is associated with adiponectin values and glycaemic disturbances. European Journal of Clinical Investigation 201343 183-189. (doi:10.1111/eci.12030)

33 Amato MC \& Giordano C. Clinical indications and proper use of visceral adiposity index. Nutrition, Metabolism, and Cardiovascular Diseases 201323 e31-e32. (doi:10.1016/j.numecd.2013.04.006)

34 Ashcroft FM \& Rorsman P. Diabetes mellitus and the $\beta$ cell: the last ten years. Cell 2012148 1160-1171. (doi:10.1016/j.cell.2012.02.010)

35 Hansen KB, Vilsboll T, Bagger JI, Holst JJ \& Knop FK. Reduced glucose tolerance and insulin resistance induced by steroid treatment, relative physical inactivity and high-calorie diet impairs the incretin effect in healthy subjects. Journal of Clinical Endocrinology and Metabolism 2010 95 3309-3317. (doi:10.1210/jc.2010-0119)

36 Khan A, Ostenson CG, Berggren PO \& Efendic S. Glucocorticoid increases glucose cycling and inhibits insulin release in pancreatic islets of ob/ob mice. American Journal of Physiology 1992263 E663-E666.

37 Catargi B, Rigalleau V, Poussin A, Ronci-Chaix N, Bex V, Vergnot V, Gin H, Roger P \& Tabarin A. Occult Cushing's syndrome in type-2 diabetes. Journal of Clinical Endocrinology and Metabolism $2003 \mathbf{8 8}$ 5808-5813. (doi:10.1210/jc.2003-030254)

38 Reimondo G, Pia A, Allasino B, Tassone F, Bovio S, Borretta G, Angeli A $\&$ Terzolo M. Screening of Cushing's syndrome in adult patients with newly diagnosed diabetes mellitus. Clinical Endocrinology 200767 225-229. (doi:10.1111/j.1365-2265.2007.02865.x)

39 Trementino L, Apolloni G, Concettoni C, Cardinaletti M, Boscaro M \& Arnaldi G. Association of glucocorticoid receptor polymorphism A3669G with decreased risk of developing diabetes in patients with Cushing's syndrome. European Journal of Endocrinology 2012166 35-42. (doi:10.1530/EJE-11-0722)

40 Munir A \& Newell-Price J. Management of diabetes mellitus in Cushing's syndrome. Neuroendocrinology 201092 (Suppl 1) 82-85. (doi:10.1159/000314316)

41 Henry RR, Ciaraldi TP, Armstrong D, Burke P, Ligueros-Saylan M \& Mudaliar S. Hyperglycaemia associated with pasireotide: results from a mechanistic study in healthy volunteers. Journal of Clinical Endocrinology and Metabolism 201398 3446-3453. (doi:10.1210/ jc.2013-1771)

42 Hiustis N, Rosen ED \& Lander ES. Reactive oxygen species have a casual role in multiple forms of insulin resistance. Nature $2006 \mathbf{4 4 0} 944-948$. (doi:10.1038/nature04634)
Received 17 September 2013

Revised version received 15 November 2013

Accepted 19 November 2013 